\title{
A Novel Bioabsorbable Sheet That Delivers NF- $\kappa$ B Decoy Oligonucleotide Restrains Abdominal Aortic Aneurysm Development in Rats
}

\author{
Shouta Akimoto, ${ }^{1}$ BS, Jun-ichi Suzuki, ${ }^{1} \mathrm{MD}$, Norio Aoyama, ${ }^{2}$ DDS, Ryota Ikeuchi, ${ }^{3} \mathrm{MS}$, \\ Hajime Watanabe, ${ }^{3}$ BS, Hiroyuki Tsujimoto,${ }^{4} \mathrm{PhD}$, Kouji Wakayama, ${ }^{1} \mathrm{MD}$, Hidetoshi Kumagai, ${ }^{1} \mathrm{PhD}$, \\ Yuichi Ikeda, ${ }^{5} \mathrm{MD}$, Hiroshi Akazawa, ${ }^{5} \mathrm{MD}$ and Issei Komuro, ${ }^{5} \mathrm{MD}$
}

\begin{abstract}
Summary
For the suppression of inflammation in the aneurysm development, we focused on inhibition of an important transcription factor, nuclear factor-kappa B (NF-KB), using a decoy strategy. We newly developed a novel bioabsorbable sheet that delivers NF- $\mathrm{KB}$ decoy oligodeoxynucleotide (ODN).

We treated 5-week-old SD rats that were induced with abdominal aortic aneurysm (AAA) using $0.5 \mathrm{M}$ $\mathrm{CaCl}_{2}$ with an NF- $\mathrm{kB}$ decoy sheet. Four weeks after AAA induction, aortic tissue was excised for further examinations. We showed that this bioabsorbable sheet could deliver the decoy ODN into the target tissues and dissolve within a week. Treatment with the NF- $\mathrm{KB}$ decoy sheet reduced the aneurysm size compared with the controls. It also suppressed inflammation due to the effect of NF- $\mathrm{KB}$ decoy ODN. Immunohistochemistry revealed that the expression of $\mathrm{CD} 31, \mathrm{CD} 4$, and $\mathrm{CD} 11 \mathrm{~b}$ in the NF-KB decoy sheet group was significantly lower than in the control sheet group. The NF- KB decoy sheet was absorbed on the target tissue.

We have revealed that the bioabsorbable sheet mediated decoy ODN is effective for transfection into target organs. We have also indicated that NF-KB decoy ODN transfection using this sheet has the potential to suppress the dilatation of aneurysm. The bioabsorbable sheet mediated transfection of the decoy ODN can be beneficial for the clinical treatment of AAA and other NF-KB-related cardiovascular diseases.
\end{abstract}

Key words: Decoy oligodeoxynucleotide

(Int Heart J 2018; 59: 1134-1141)

A bdominal aortic aneurysm (AAA) is a common degenerative condition with high mortality in humans. It is common in males over 50 years old, and smoking, obesity, and hypertension are recognized as risk factors. Elective surgical or endovascular repair is performed to prevent rupture of large AAAs, whereas some of them could not rescue the patients. ${ }^{1-3)}$ Therefore, new methodology for prevention and treatments of AAA is required. It has been reported that chronic inflammation is one of the causes of AAA and aortic dilatation. ${ }^{4-6)} \mathrm{Nu}-$ clear factor-kappa B (NF-KB) has an activity to control this inflammation. NF- $\mathrm{KB}$, which receives stimuli such as cytokines and oxidative stress response, is known to cause cell proliferation and inflammation. Matrix metalloproteases (MMPs), a kind of proteolytic enzyme, play an important role in the pathogenesis of AAAs. ${ }^{1,7,8)}$ Especially, it is well known that elastin of blood vessels is degraded by the occurrence of MMP-2 and MMP-9. The activity of
MMP leads to damage of the blood vessel wall and abnormal extension of the blood vessel diameter. ${ }^{9,10)} \mathrm{NF}-\mathrm{KB}$ can also promote chronic inflammation in the aortic wall and regulate MMPs transcription. In human and animal experiments, inhibition of NF- $\mathrm{KB}$ activation can prevent the development of AAA. ${ }^{11,12}$ Thus, it has been attracting attention as a therapeutic target.

Recently, the NF- $\kappa B$ decoy oligodeoxynucleotide $(\mathrm{ODN})$, which is a nucleic acid drug, has been reported to have an anti-inflammatory effect. NF- $\mathrm{KB}$ decoy ODN is a double-stranded DNA with specific affinity to the cis-

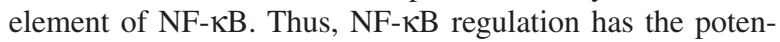
tial to suppress the AAA progression. The decoy ODN suppresses many inflammatory factors including adhesion molecules, cytokines, and chemokines. ${ }^{13)}$ Decoy ODN transfection is a significant methodology for suppressing gene activation. Clinically, we have reported that NF-KB decoy ODN transfection to the coronary artery might pre-

From the ${ }^{1}$ Department of Advanced Clinical Science and Therapeutics, School of Medicine, The University of Tokyo, Tokyo, Japan, ${ }^{2}$ Department of Oral Interdisciplinary Medicine, Kanagawa Dental University, Kanagawa, Japan, ${ }^{3}$ AnGes, Inc., Osaka, Japan, ${ }^{4}$ Hosokawa Micron Co., Osaka, Japan and ${ }^{5}$ Department of Cardiovascular Medicine, School of Medicine, The University of Tokyo, Tokyo, Japan.

This study was supported by grants from the Medical Pharmaceutical Research Support Fugaku Foundation, the Japan Heart Foundation, and the Takeda Research Foundation.

Address for correspondence: Jun-ichi Suzuki, MD, Department of Advanced Clinical Science and Therapeutics, School of Medicine, The University of Tokyo, 7-3-1 Hongo, Bunkyo, Tokyo, 113-8655, Japan. E-mail: junichisuzuki-circ@umin.ac.jp

Received for publication November 7, 2017. Revised and accepted November 28, 2017.

Released in advance online on J-STAGE August 11, 2018.

doi: 10.1536/ihj.17-632

All rights reserved by the International Heart Journal Association. 
vent restenosis. ${ }^{14}$ Alternatively, the effect of the NF- $\kappa B$ decoy ODN from the balloon catheters has been reported; however, the transfection and effectiveness of NF- $\mathrm{KB}$ decoy ODN using a bioabsorbable sheet has not yet been demonstrated. ${ }^{15}$ Recently, we demonstrated that the chimeric decoy ODN strategy against both NF- $\kappa \mathrm{B}$ and ets (E26 transformation-specific sequence) might provide a new therapeutic strategy to treat AAA. At that time, we used a cellulose-based delivery sheet containing chimeric decoy ODN. Although the technique was minimally invasive, it may include pathological problems. Because the cellulose-based delivery sheet remains on the aorta for long time, it may cause unexpected immunological reactions. Thus, we developed a bioabsorbable sheet that automatically dissolves after the delivery of decoy ODN. This might be ideal to avoid possible adverse effects in clinical settings. In this study, we examined the effect NF- $\kappa \mathrm{B}$ decoy ODN which was delivered from a novel bioabsorbable sheet on AAA.

\section{Methods}

Animals: This study used male Sprague-Dawley rats (4 weeks old, CLEA Japan, Inc.). The handling of laboratory animals and their use in the experiments conformed to the Guidelines for Animal Experiments at the University of Tokyo. The rats were maintained in cages ( 2 per cage) in a temperature- and humidity-controlled condition with a 12 hour light/12 hour dark cycle. Rats were allowed free access to standard food and water throughout the experiments.

Bioabsorbable sheet-mediated transfection of FITClabeled decoy ODN into abdominal aorta: We newly developed a novel bioabsorbable sheet with the JP patent P5996990 (02, September, 2016). To study the in vivo transfection efficiency of decoy ODN using a bioabsorbable sheet, we used the FITC-labeled decoy ODN and the rat AAA model. The decoy ODN was made (Fasmac Co. Ltd, Kanagawa, Japan), and FITC was labeled to 5' terminals of the decoy ODN by the manufacturer. Immediately after $\mathrm{CaCl}_{2}$ application, the bioabsorbable sheet with FITC-labeled decoy ODN was placed on the perivascular surface of the aorta. For control studies, the bioabsorbable sheet without FITC-labeled decoy ODN was attached on the aorta. The aortas were harvested on day 7; the transfection efficiency was judged using fluorescent microscopy. ${ }^{15,16)}$ To show the unexpected dissemination of the decoy ODN, we also harvested other organs (liver and kidney) which the bioabsorbable sheet was not applied to.

AAA model: The rats were anesthetized by intraperitoneal injection with $3.6 \%$ chloral hydrate $(100 \mathrm{~g} / \mathrm{mL}$, Nacalai tesque Inc., Japan) and then disinfected by shearing in a supine position. An incision was made on the abdominal median, and the abdominal cavity was exposed. Following this, the abdominal aorta below the renal artery and above the iliac artery was dissociated out, it was covered by gauze containing $0.5 \mathrm{M} \mathrm{CaCl} \mathrm{Cl}_{2}$ solution or saline and stimulated for 15 minutes. 15 minutes later, saline covering the abdominal aorta and gauze collected $0.5 \mathrm{M} \mathrm{CaCl}_{2}$ were removed and the peritoneal cavity was carefully washed three times with saline. ${ }^{17)}$ The incision was sutured layer by layer. Four weeks after the operation, the animals were anesthetized, and the abdominal aorta was isolated.

Treatment with the bioabsorbable NF- $\kappa$ B decoy sheet: The sheet was soaked in saline and then attached to the surface of the abdominal aorta just after washing the abdominal aorta. To fix the decoy sheet, Neoveil (Gunze Inc., Japan) was attached over the decoy sheet. Neoveil, which is widely used in clinical operation, is also bioabsorbable on the target organs. Sequences of the phosphorothioate ODN used in this study were as follows: NF$\kappa \mathrm{B}$ decoy ODN (consensus sequences are underlined), 5'CCTTGAAGGGATTTCCCTCC-3' and 3'-GGAACTTCC CTAAAGGGAGG-5'; and scrambled decoy ODN, 5'-TT GCCGTACCTGACTTAGCC-3' and 3'-AACGGCATGGA CTGAATCGG-5, ${ }^{13,15,18)}$

Experimental groups: We made 5 experimental groups; saline + control sheet, saline $+\mathrm{NF}-\kappa \mathrm{B}$ decoy sheet, $\mathrm{CaCl}_{2}$ + control sheet, $\mathrm{CaCl}_{2}+\mathrm{NF}-\kappa \mathrm{B}$ sheet, and saline + FITClabeled decoy sheet.

Macroscopic assessments: The diameter of the artery was measured on day 28. The animals were anesthetized, and the abdominal aorta was exposed. Photos of incorporation were taken using OLYMPUS digital camera STYLUS TG-4 Tough. The aortic diameter was measured and calculated. We confirmed whether the NF- $\kappa \mathrm{B}$ decoy sheet was absorbed or not macroscopically.

Transfection of FITC-labeled decoy ODN assessment in aorta: We sacrificed rats treated with a FITC-labeled decoy oligonucleotide decoy sheet 7 days later. Transfection of FITC-labeled decoy ODN was analyzed under a fluorescence microscope. Our preliminary data showed that this sheet delivered FITC-labeled ODN in the aortic wall and that the signal lasted at least 4 weeks.

Measurement of aortic diameter: Pathological specimens of hematoxylin-eosin (HE) staining were taken, and the diameter length of the aorta was measured with ImageJ and compared with each experimental group as follows. We traced the aortic perimeter using the software and divided it with the ratio of the circumference of a circle to its diameter.

Histology: We perfused with PBS systemically just before the organ harvesting. For histological analysis, abdominal aorta samples fixed with $10 \%$ formaldehyde neutral buffer solution (Nacalai tesque Inc., Japan) were embedded in paraffin, and $5 \mu \mathrm{m}$ thick sections were stained with $\mathrm{HE}$ and elastica van Gieson (EVG). Evaluation was performed using architecture scores to evaluate elastin in the aortic wall. The elastic fiber integrity was assessed in four representative areas on a scale from 0 to 3 , with scoring as follows: 0 , completely intact with wavy organization; 1 , mild disorganization without fragmentation; 2, local degradation and fragmentation; and 3, extensive fragmentation and degradation. ${ }^{18)}$

Immunofluorescence staining: The extracted rat aortas were fixed embedded in O.T.C. compound (Sakura tissue tek Jpapan) at $-85 \mathrm{C}$, and sectioned at $8 \mu \mathrm{m}$ by Cryostat (Leica). Next, we washed with PBS after drying at room temperature and incubated with 5\% NGSPBSt for 1 hour at room temperature. After blocking with 5\% NGSPBSt, the primary antibody was incubated overnight. We diluted mouse anti-CD31 Pcam-1 H-3: sc-376764 (1:200, Santa 


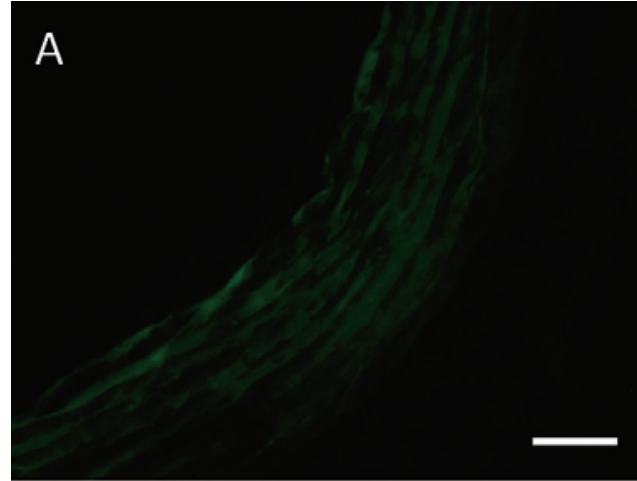

Saline

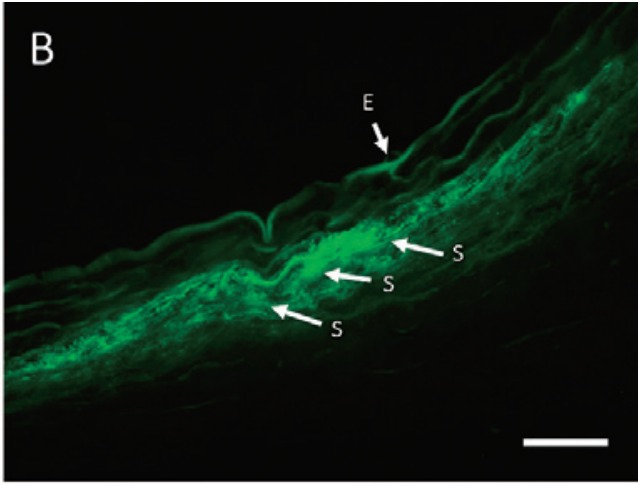

FITC ODN

Figure 1. Transfection efficiency of FITC decoy for abdominal aorta. Fluorescence images of native aorta (A) and transfected FITC decoy ODN (B) are shown. The arrows in panel B indicate FITC-labeled decoy transfected smooth muscle $(\mathrm{S})$ and endothelial $(\mathrm{E})$ cells. Scale bars $=50 \mu \mathrm{m}$.

Cruz Biotechnology, Inc., Santa Cruz, California, USA), mouse anti-CD4 W3/25 (1:200, GeneTex), and CD11b (1: 200, PharMingen, San Diego, CA) with $5 \%$ NGS in PBSt as the primary antibody. After the secondary antibody added, we incubated them for 1 hour at room temperature. We graded the intensity semi-quantitatively: 0 , no staining; 1 , very mild staining; 2 , mild staining; 3 , moderate staining; and 4, abundant positive staining. ${ }^{19,20)}$

Statistical analysis: Values are given as mean \pm SE. We used Student's $t$-test for comparisons between the 2 groups. Differences were considered statistically significant at a value of $P<0.05$.

\section{Results}

The bioabsorbable sheet transfected FITC-labeled decoy ODN into the target aortas: Seven days after, Seven days after affixing the sheet. the FITC labeled decoy ODN sheet treated group showed more intense fluorescence in the endothelial and medial smooth muscle cells in the aortic wall (Figure 1). The decoy ODN was not transfected into other organs where the bioabsorbable sheet was not applied (data not shown). No systemic adverse effect was observed in the rats. Absorption of the decoy sheet and Neoveil was also confirmed using macroscopic observation on day 7 (Figure 2).

Diameter of abdominal aorta: We measured the aortic diameter in the AAA group, and it was enlarged compared with the Saline group. However, the diameter of the AAA + decoy sheet group was significantly suppressed compared with the AAA group (Figures 3, 4).

Microscopic assessment: We used samples with HE (Figure 5) and EVG (Figure 6) staining to observe inflammatory cells and elastic fibers in each group. Intensive inflammatory cell infiltration was observed in the AAA group compared with the control group. It is noteworthy that inflammatory cells were barely observed in the AAA + decoy sheet group. Although the elastic fibers showed a wavy structure in the control group, the elastic fiber in the AAA group had a flat structure and the endothelial structure failed. Although the elastic fibers showed a wavy structure in the control group, the elastic fiber in the AAA group had a flat structure and the endothelial structure failed. In the evaluation of the aortic wall at the architectural score, the decoy sheet treated group had a lower score than the AAA group $(P<0.05)$ (Figure 7 ).

NF-kB decoy ODN transfection with the bioabsorbable sheet suppressed expression of inflammatory factors: To clarify the mechanism, we performed immunohistochemistry. Expression of CD31, CD4, and CD11b was intensely enhanced in the aortic walls in the control group, whereas expression was reduced in the aortas from rats that received the NF- $\mathrm{KB}$ decoy ODN with the bioabsorbable sheet. Very faint and limited expression of these factors was detected in native aortas and aortas from shamoperated rats (Figure 8). The NF- $\mathrm{BB}$ decoy ODN with the bioabsorbable sheet significantly suppressed the intensity of CD31 and CD11b compared with non-treated $\mathrm{CaCl}_{2}$ group (Figure 9).

\section{Discussion}

In this study, we demonstrated the significant effect

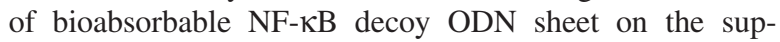
pression of AAA development and the degradation of the elastin structure. This study revealed that treatment with AAA can be performed with a single nucleic acid drug treatment.

Firstly, we showed that the bioabsorbable sheet enhanced the transfection efficiency of the decoy ODN. Previous studies have indicated that the therapeutic application of decoy ODN is largely dependent on the development of a delivery vehicle that can efficiently deliver the decoy ODN to target cells. ${ }^{21)}$ As we have shown in this study, the bioabsorbable sheet method can deliver decoy ODN into the target aortas. This might prevent systemic adverse effects because the decoy ODN was not transfected into other organs which bioabsorbable sheet was not applied. This bioabsorbable sheet could be applicable for other delivery systems such as a cellulose-based delivery sheet, because this sheet dissolved within a week. Therefore, it may prevent adverse immunological reaction 


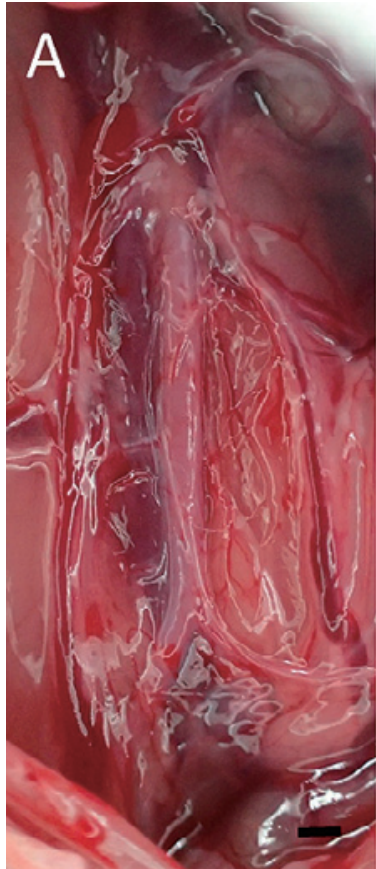

Day 0

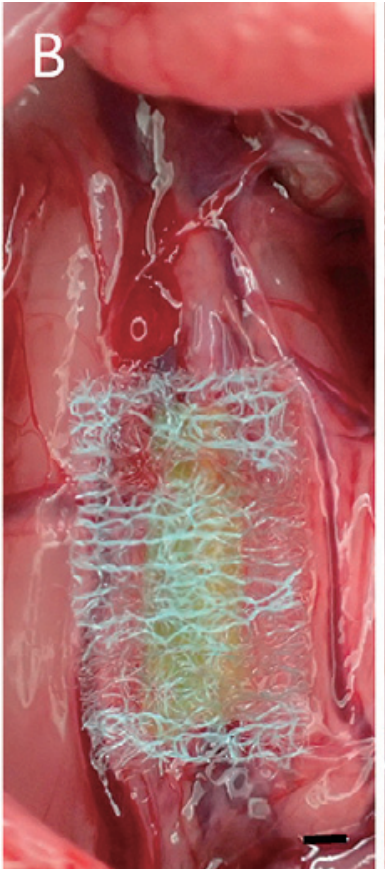

Treatment decoy sheet

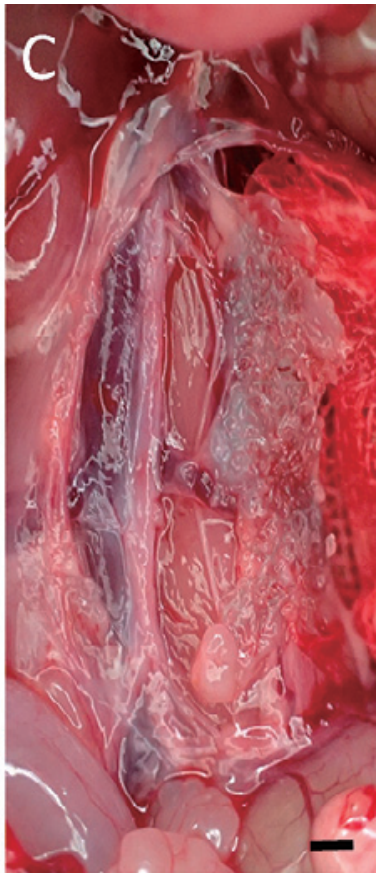

Day 7

Figure 2. Macroscopic photos of bioabsorbable sheet on abdominal aorta. The panels show the macroscopic image of abdominal aorta before NF- $\mathrm{KB}$ decoy sheet applied on day $0(\mathbf{A})$, just adhered the sheet on day $0(\mathbf{B})$, and day 7 (C). We confirmed that the sheet was completely absorbed on day 7 . Scale bars $=1 \mathrm{~mm}$.

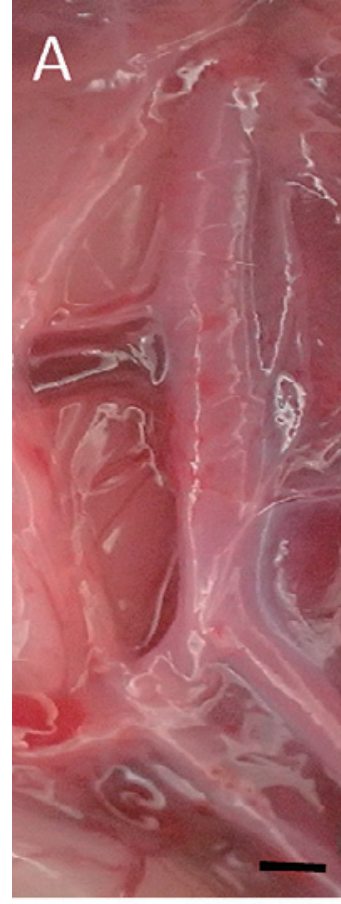

Saline

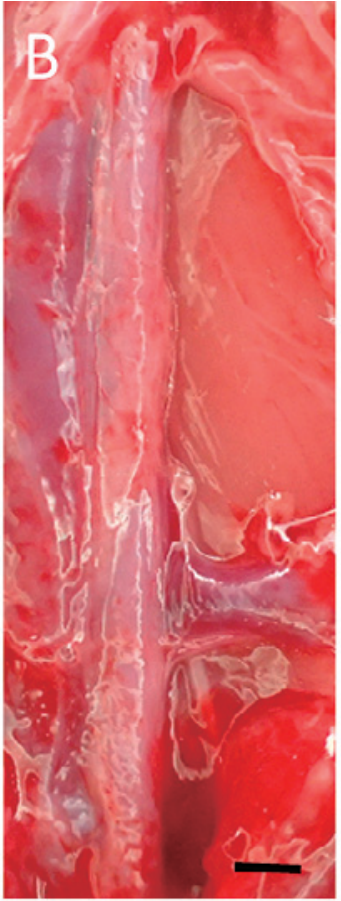

Saline + Decoy Sheet

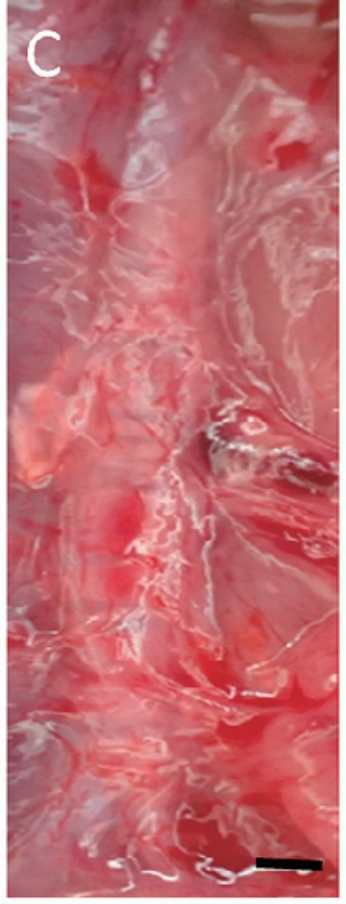

$\mathrm{CaCl} 2$

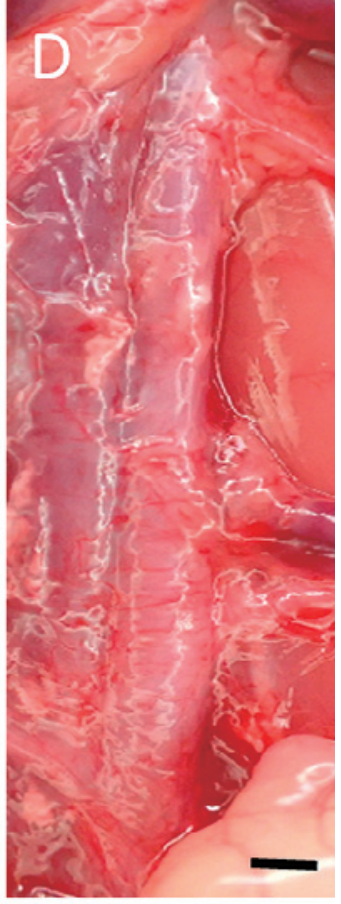

$\mathrm{CaCl} 2+$ Decoy Sheet

Figure 3. Representative macroscopic findings of AAA. The panels show the macroscopic findings of the abdominal aorta on day 28. We performed measurements using HE-stained samples. The diameter of the artery in the AAA group $(n=5)$ was enlarged compared with the Saline group $(n=5)$. However, the diameter of the AAA + decoy sheet group $(n=5)$ suppressed compared with the AAA group $(n=5)$. Scale bars $=1 \mathrm{~mm}$. 


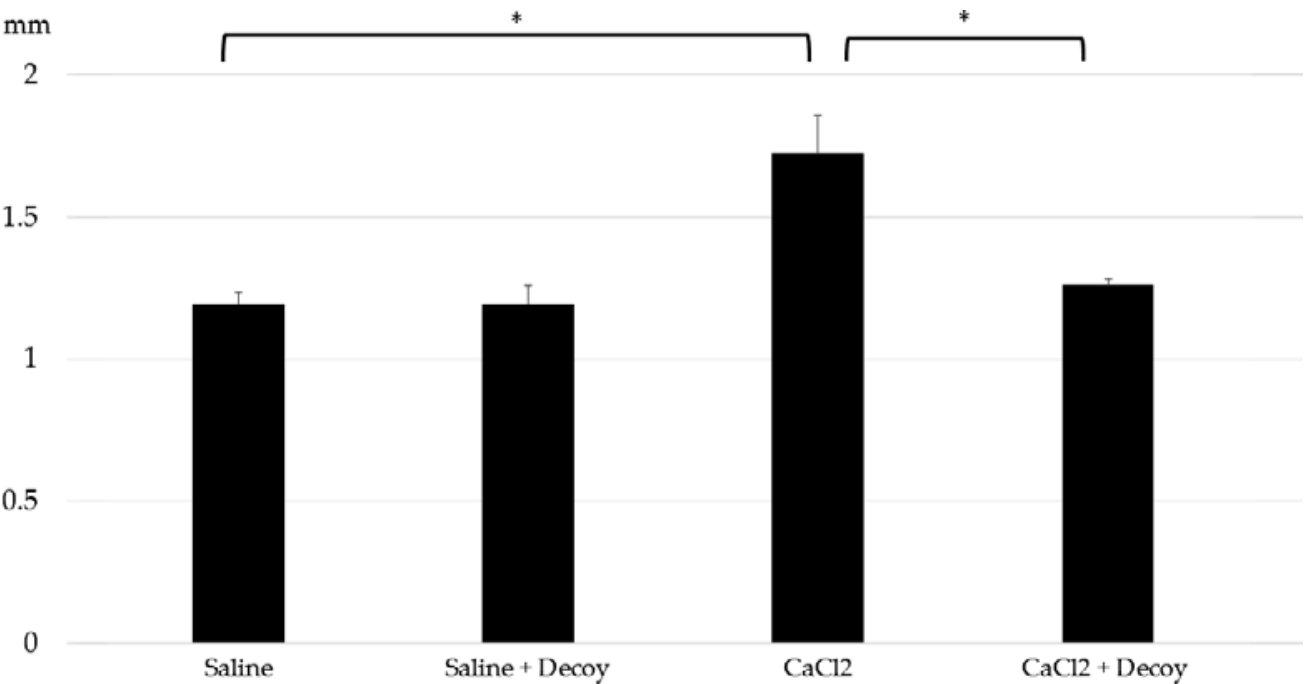

Figure 4. Quantitative results of aortic diameter. The graph shows the quantitative results of aortic diameter on day 28
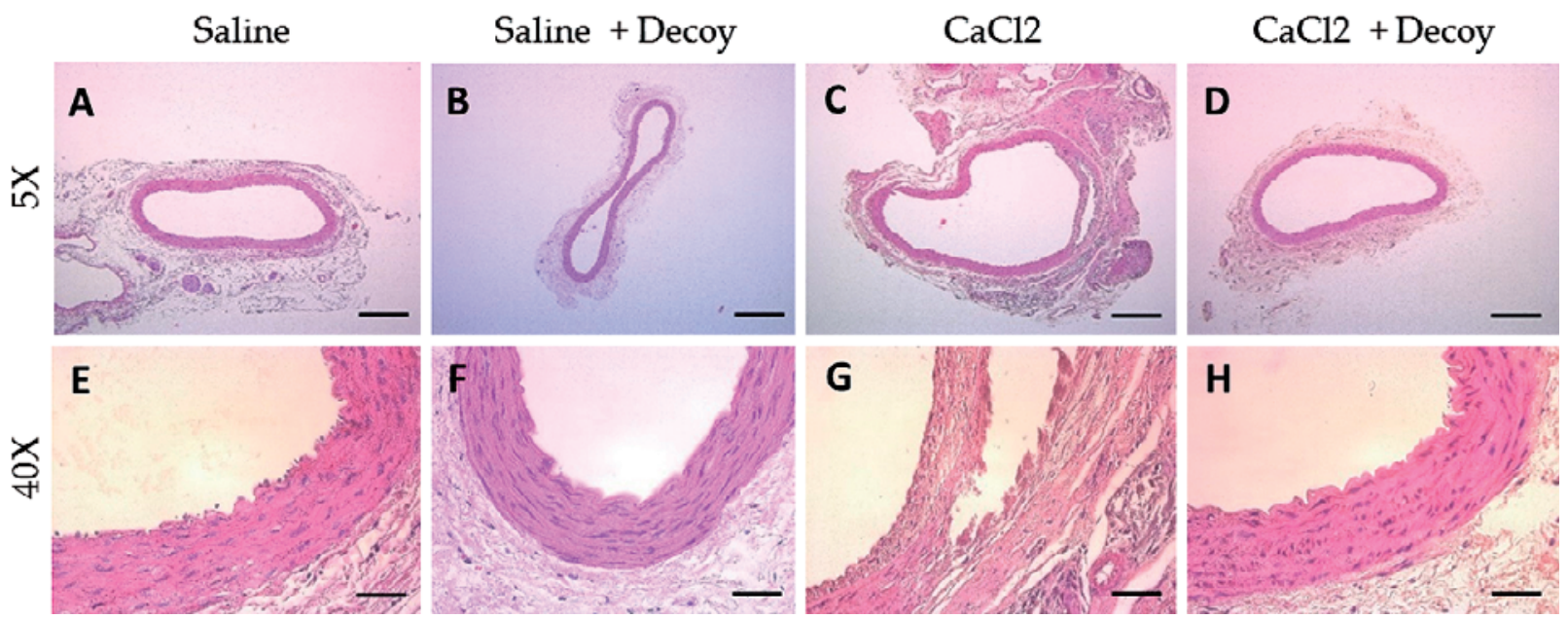

Figure 5. Representative microscopic HE staining. Panels show the microscopic findings of the aorta with HE staining; saline (A and $\mathbf{E})$, saline $+\mathrm{NF}-\mathrm{\kappa B}$ decoy sheet $(\mathbf{B}$ and $\mathbf{F}), \mathrm{CaCl}_{2}(\mathbf{C}$ and $\mathbf{G})$, and $\mathrm{CaCl}_{2}+\mathrm{NF}-\kappa \mathrm{B}$ decoy sheet $(\mathbf{D}$ and $\mathbf{H})$. Scale bars indicate $400 \mu \mathrm{m}$ in upper panels and 50 $\mu \mathrm{m}$ in the lower panels.
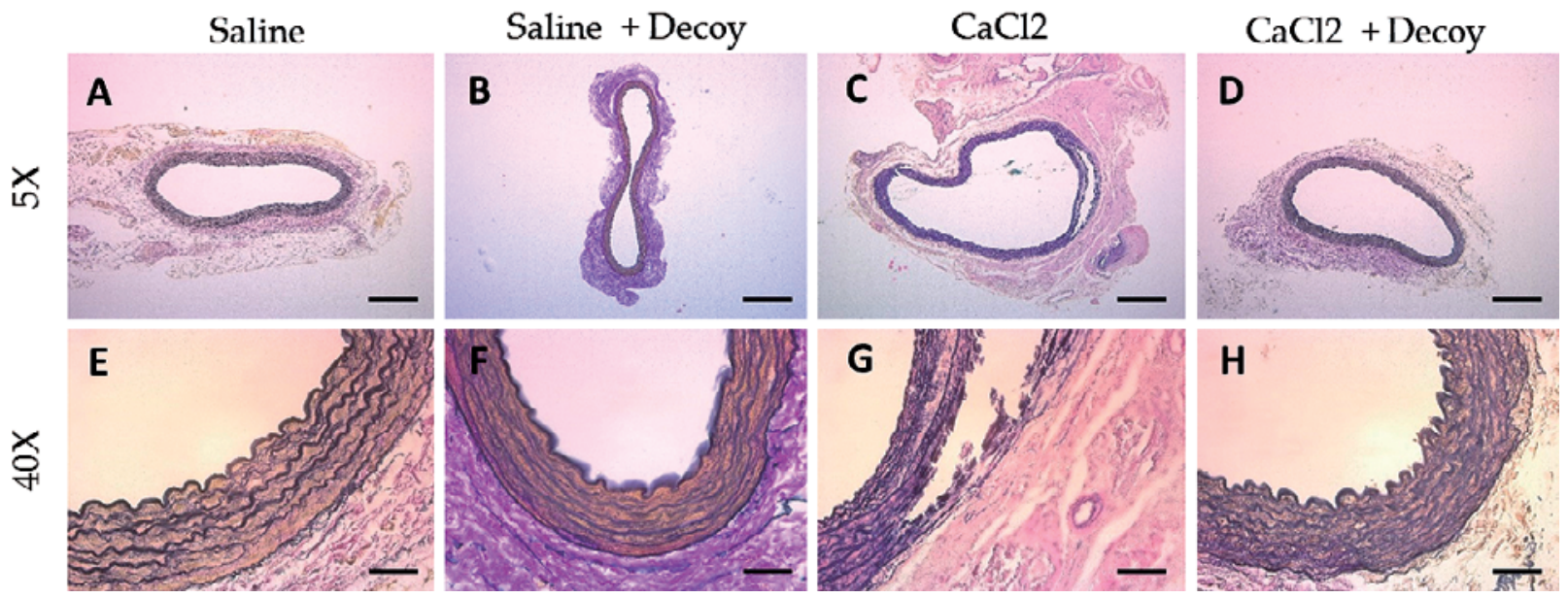

Figure 6. Representative EVG staining findings. Panels show the microscopic findings of the aorta with EVG staining; saline (A and $\mathbf{E})$, saline + NF- $\kappa B$ decoy sheet $(\mathbf{B}$ and $\mathbf{F}), \mathrm{CaCl}_{2}(\mathbf{C}$ and $\mathbf{G})$, and $\mathrm{CaCl}_{2}+\mathrm{NF}-\kappa \mathrm{B}$ decoy sheet $(\mathbf{D}$ and $\mathbf{H})$. Scale bars indicate $400 \mu \mathrm{m}$ in upper panels and $50 \mu \mathrm{m}$ in the lower panels. 


\section{Scores}

3

2.5

2

1.5

1

0.5

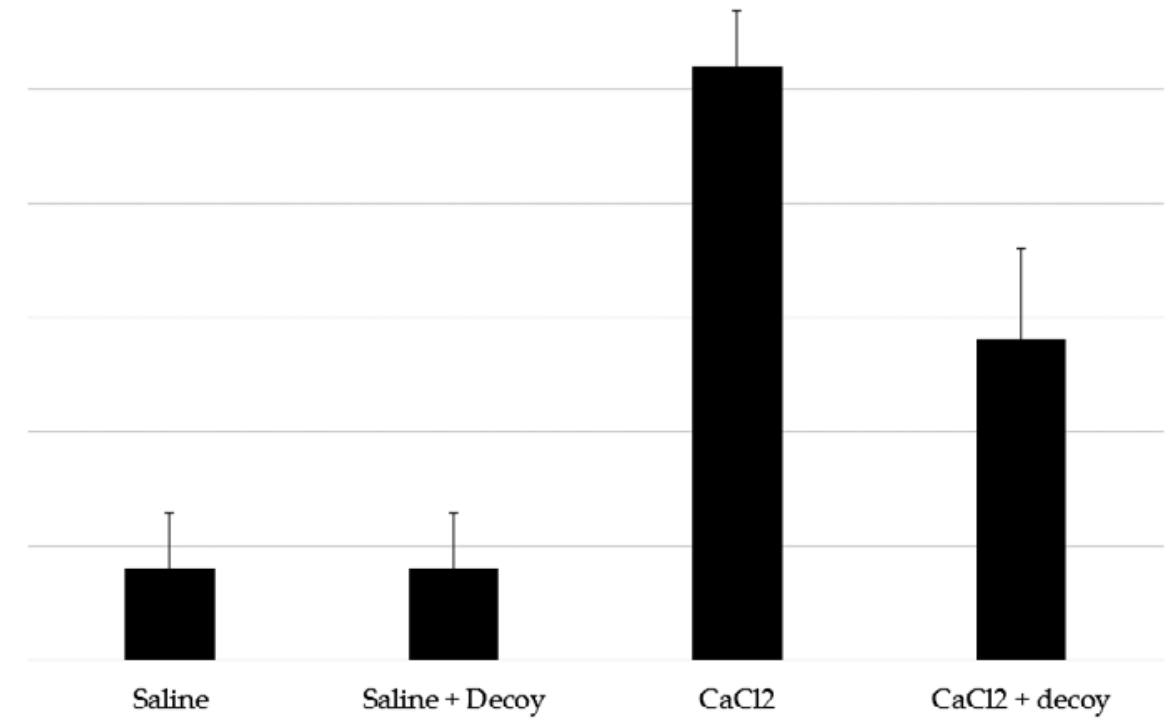

Figure 7. Quantitative results of architecture scores of the abdominal aorta. The graph shows the results of architecture scores of the aortic wall. $(n=5$ each).
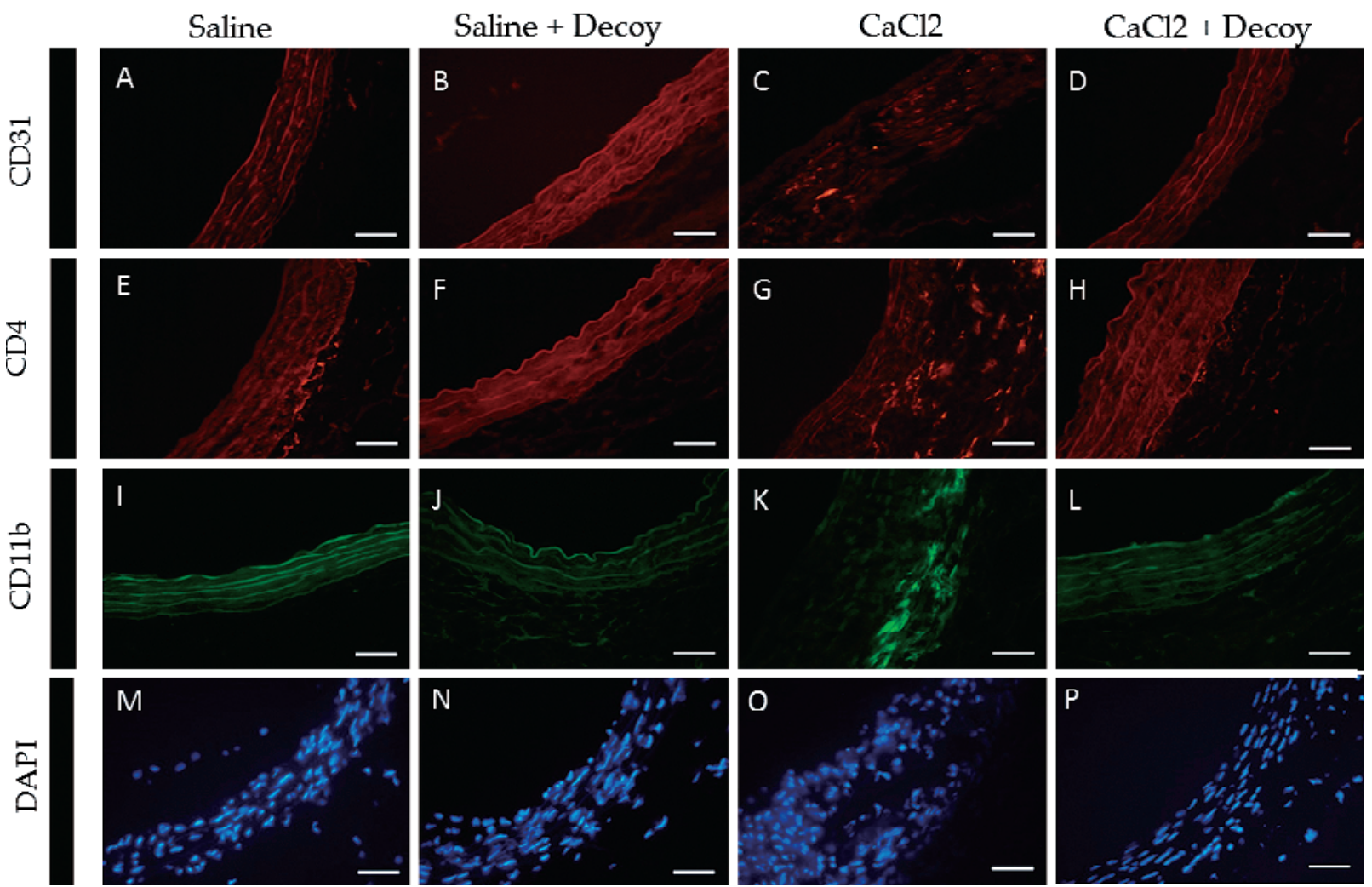

Figure 8. Immunohistochemical staining for CD31, CD4, and CD11b. Representative results of immunohistochemical staining for CD31 (A-D), CD4 (E-H), and CD11b (I-L) of aortic walls on day 28 are shown. We add nuclear stain with DAPI (4',6-diamidino-2-phenylindole) staining (M-

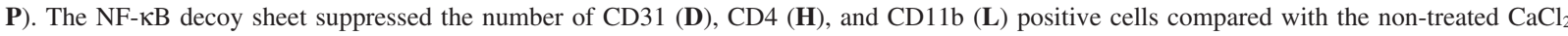
group $(\mathbf{C}, \mathbf{G}$, and $\mathbf{K})$. Scale bars $=50 \mu \mathrm{m}$ 

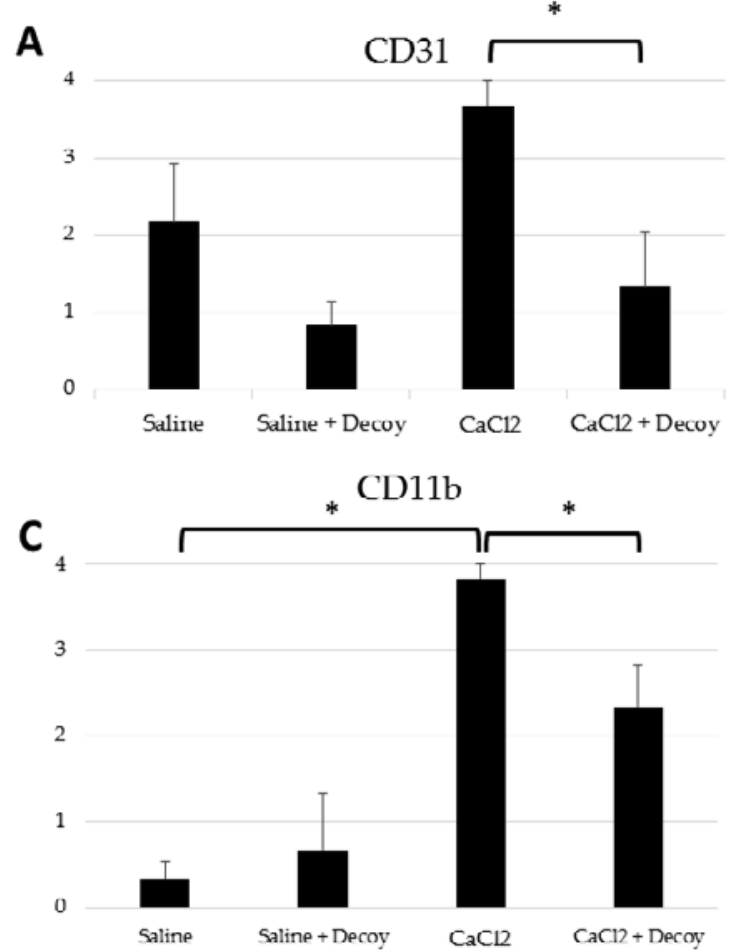

B

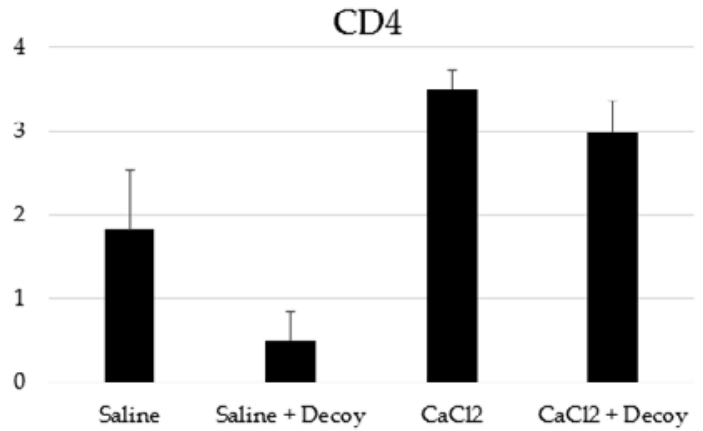

Figure 9. Immunofluorescent intensity. These bar graphs show immunofluorescent intensity of CD31 (A), CD4 (B), and CD11b (C). The NF$\kappa \mathrm{B}$ decoy $\mathrm{ODN}$ with the bioabsorbable sheet significantly suppressed the intensity of CD31 and $\mathrm{CD} 11 \mathrm{~b}$ compared with non-treated CaCl $\mathrm{C}_{2}$ group. However, the treatment did not suppress CD4 intensity, statistically.

caused by remaining materials. These results also indicate that it is possible to perform local gene delivery. Thus, the bioabsorbable sheet is a novel, nonviral, effective, and safe method of in vivo gene transfer.

Using this methodology, we have revealed that in vivo transfection of $\mathrm{NF}-\kappa \mathrm{B}$ decoy ODN significantly suppressed the development of AAA and enhancement of inflammatory factors without systemic adverse effects. Inflammation is one of the main causes of AAA; thus, we used a rat model of AAA induced by $\mathrm{CaCl}_{2}$. NF- $\kappa \mathrm{B}$ plays a central role in the progression of inflammation in cardiovascular diseases. ${ }^{22)}$ Immune modulation of NF- $\mathrm{KB}$ using decoy ODN could be clinically useful for the prevention of aortic wall degradation during AAA development and other inflammatory cardiovascular diseases without systemic immune modulation and adverse effects. These results point out new drug treatments for AAA.

In conclusion, the bioabsorbable sheet effectively transfected the decoy ODN into target organs in vivo. Using this method, NF- $\mathrm{KB}$ decoy ODN has the potential to suppress aortic wall destruction during AAA development. Thus, transfection of NF- $\mathrm{\kappa B}$ decoy ODN using a bioabsorbable sheet may be beneficial for the clinical treatment of cardiovascular and other inflammatory diseases.

\section{Disclosures}

Conflicts of interest: None.

\section{References}

1. Limet R, Defawe OD. Abdominal aortic aneurysm. Lancet 2005; 365: 1577-89.

2. Miyake T, Morishita R. Pharmacological treatment of abdominal aortic aneurysm. Cardiovasc Res 2009; 83: 436-43.

3. Zhang R, Chen S, Zhang H, et al. Biomarkers investigation for in-hospital death in patients with Stanford type A acute aortic dissection. Int Heart J 2016; 57: 622-6.

4. Treska V, Kocova J, Boudova L, et al. Inflammation in the wall of abdominal aortic aneurysm and its role in the symptomatology of aneurysm. Cytokines Cell Mol Ther 2002; 7: 91-7.

5. Daugherty A, Cassis LA. Mechanisms of abdominal aortic aneurysm formation. Curr Atheroscler Rep 2002; 4: 222-7.

6. Suzuki J, Imai Y, Aoki M, et al. Periodontitis may deteriorate sinus of valsalva dilatation in Marfan syndrome patients. Int Heart J 2016; 57: 456-60.

7. Chase AJ, Bond M, Crook MF, Newby AC. Role of nuclear factor-kappa B activation in metalloproteinase-1, -3 , and -9 secretion by human macrophages in vitro and rabbit foam cells produced in vivo. Arterioscler Thromb Vasc Biol 2002; 22: 76571.

8. Li J, Krishna SM, Golledge J. The potential role of kallistatin in the development of abdominal aortic aneurysm. Int J Mol Sci 2016; 11: 17.

9. Newman KM, Ogata Y, Malon AM, et al. Identification of matrix metalloproteinases 3 (stromelysin-1) and 9 (gelatinase B) in abdominal aortic aneurysm. Arterioscler Thromb 1994; 14: 1315-20.

10. Thompson RW, Parks WC. Role of matrix metalloproteinases in abdominal aorticaneurysms. Ann N Y Acad Sci 1996; 800: 15774.

11. Yan YW, Fan J, Bai SL, Hou WJ, Li X, Tong H. Zinc prevents abdominal aortic aneurysm formation by induction of A20- 
mediated suppression of NF-אB pathway. PLOS ONE 2016; 11 e0148536.

12. Parodi FE, Mao D, Ennis TL, Bartoli MA, Thompson RW. Suppression of experimental abdominal aortic aneurysms in mice by treatment with pyrrolidine dithiocarbamate, an antioxidant inhibitor of nuclear factor-kappaB. J Vasc Surg 2005; 41: 47989.

13. Suzuki J, Morishita R, Amano J, Kaneda Y, Isobe M. Decoy against nuclear factor-kappa B attenuates myocardial cell infiltration and arterial neointimal formation in murine cardiac allografts. Gene Ther 2000; 7: 1847-52.

14. Suzuki J, Ito $\mathrm{H}$, Gotoh R, Morishita R, Egashira K, Isobe M. Initial clinical cases using an NF-kB decoy ODN at the site of the coronary stenting for prevention of restenosis. Circ J 2004; 68: 270-1.

15. Miyake T, Ihara S, Miyake T, et al. Prevention of neointimal formation after angioplasty using nuclear factor- $\mathrm{\kappa B}$ decoy oligo eoxynucleotide-coated balloon catheter in rabbit model. Circ Cardiovasc Interv 2014; 7: 787-96.

16. Miyake T, Aoki M, Osako MK, Shimamura M, Nakagami H, Morishita R. Systemic administration of ribbon-type decoy oligodeoxynucleotide against nuclear factor $\mathrm{\kappa B}$ and ets prevents abdominal aortic aneurysm in rat model. Mol Ther 2011; 19:
181-7.

17. Wang Y, Krishna S, Golledge J. The calcium chloride-induced rodent model of abdominal aortic aneurysm. Atherosclerosis 2013; 226: 29-39.

18. Nakashima H, Aoki M, Miyake T, et al. Inhibition of experimental abdominal aortic aneurysm in the rat by use of decoy oligodeoxynucleotides suppressing activity of nuclear factor kappaB and ets transcription factors. Circulation 2004; 109: $132-8$.

19. Aoyama N, Suzuki JI, Ogawa M, et al. Clarithromycin suppresses the periodontal bacteria-accelerated abdominal aortic aneurysms in mice. J Periodont Res 2012; 47: 463-9.

20. Aoyama N, Suzuki J, Ogawa M, et al. Toll-like receptor-2 plays a fundamental role in periodontal bacteria-accelerated abdominal aortic aneurysms. Circ J 2013; 77: 1565-73.

21. Futamatsu H, Suzuki J, Kosuge H, et al. Attenuation of experimental autoimmune myocarditis by blocking activated $\mathrm{T}$ cells through inducible costimulatory molecule pathway. Cardiovasc Res 2003; 59: 95-104.

22. Morishita R, Tomita N, Kaneda Y, Ogihara T. Molecular therapy to inhibit NFkappaB activation by transcription factor decoy ODN oligonucleotides. Curr Opin Pharmacol 2004; 4: 139-46. 